\title{
When internal and emergency medicine speak to each other: organization in the time of COVID
}

\author{
Chiara Cogliati $^{1}$. Elisa Ceriani ${ }^{1}$ (D) $\cdot$ Anna Maria Brambilla ${ }^{2}$
}

Received: 30 April 2020 / Accepted: 14 May 2020 / Published online: 29 May 2020

(c) Società Italiana di Medicina Interna (SIMI) 2020

\section{Dear Editor,}

During the night of 20-21 February 2020, the first case of domestic SARS-CoV-2 (severe acute respiratory syndrome coronavirus 2) was diagnosed in Northern Italy, in the Lombardy region. Later, the coronavirus outbreak spread throughout the entire region [1]. Hospitals involved in the epidemic needed to change their internal organization and "modus operandi" in order to accommodate the growing number of affected patients. We would like to report and share our experience, which involved the Emergency and the Internal Medicine Departments, particularly addressing the setting of medium-intensity care wards for hospitalized Covid patients.

Luigi Sacco Hospital is a medium sized (600 beds), University-Affiliated Hospital in northwest Milan. It handles almost all the main medical/surgical specialties (except for Neurosurgery), but due to the presence of a huge Infectious Diseases Department it has always been a specialty-referral center for northern Italy. For this reason, other hospitals referred the first diagnosed Covid-19 patients to our Infectious Diseases and Intensive Care Unit (ICU), as they were adequately prepared for bio-emergencies. This allowed other sectors of the hospital time to organize in case of an epidemic increase, which unfortunately occurred. The number of infected patients needing hospitalization grew rapidly. On 11 March 2020, close collaboration between the Emergency

Elisa Ceriani

elisa.ceriani@live.com

Chiara Cogliati

chiara.cogliati@fastwebnet.it

Anna Maria Brambilla

annamaria.brambilla@asst-fbf-sacco.it

1 Department of Internal Medicine, Luigi Sacco Hospital, University of Milan, Via G.B.Grassi 74, Milan, Italy

2 Department of Emergency, L. Sacco Hospital, University of Milan, Milan, Italy and the Internal Medicine Departments began. The Emergency Department staff set-up repeated 3-h training sessions on the use of continuous Positive Airway Pressure (CPAP) helmets [2], while the Department of Infectious Diseases provided multiple daily training sessions regarding the correct use of Personal Protective Equipment (PPE) [3]. Moreover, since all Internal and Emergency Medicine specialists and residents had already been adequately trained in bedside chest ultrasound, a standardized reporting protocol was shared, in order to monitor lung disease efficaciously.

We decided to create a shared staff, including consultants and residents from the Internal Medicine and the Emergency Departments, in order to facilitate the transfer of different skills between them (to allow for role flexibility in case of staff shortage) and to learn as much as possible from this rare situation. Internists completely changed their shift habits, from a model of care where they usually looked after patients from admission to discharge, to an intensive care model which assured $24 \mathrm{~h}$ intensive patients' surveillance over three major shifts. In the medical area, on 11 March, a 30-bed medium-intensity Care Unit was opened for COVID patients. The Pneumology ward was transformed into a 20-bed sub-intensive unit for the most critical patients. After a few days, since the epidemic spread continued growing, another 30-bed Unit was opened. The Internal Medicine and Emergency medicine staff was further implemented by Rheumatologists, Cardiac Surgeons, Cardiologists, and multidisciplinary teams for each shift were set. Patients were admitted to our wards either from other hospitals - in particular from Bergamo, Brescia, and east-Milano districts, the most impacted area in the Italian Covid-19 epidemic- and from our ED. Illness severity ranged from the need for oxygen support to CPAP support. Later, on 20 March, a 30-bed low-intensity care unit was opened for recovering patients with low oxygen needs or already weaned. They were transferred from the other hospital wards in order to guarantee increased bed availability for acute patients. Colleagues from the surgical area (general surgeons, orthopedists, 
otolaryngologists, ophthalmologists) took charge of these patients, with external (out of the isolation zone) supervision by senior Internal Medicine consultants. In this way, older colleagues were involved, in an effort to reduce work risk for this age group.

Difficult cases were discussed daily with infectious disease and ICU consultants. Social media were employed to share ongoing literature and the current research protocols in order to improve internal communication as shift work and infection prevention protocols do not permit frequent/ crowded meetings.

Our hospital shifted a total number of 330 beds to Covid19 patient care, including the ER, ICU, Infectious Disease, Pneumology, Internal Medicine, Obstetrics-Gynecology, Pediatrics units.

As of April 28, we had managed 276 COVID patients in our medium-intensity 60 beds. Of the total number of patients, 51 needed CPAP support. The mean age of our population was 63 years old, ranging from 15 to 95 . Thirty-six patients died (13\%) and 13 patients were transferred to ICU.

A perfect world? Definitely not, we have no supporting data nor claim that this is a flawless organization model. However, we did our best to ensure that $60+30$-bed COVID wards opened in just a few days, trying to offer an acceptable standard of care due to intense training, unexpected teamwork cooperation and the sharing of our skills and experiences. The presence of a preexisting Bio-emergency Unit, the mid-size of our hospital and collaborative General Direction ensured the possibility to rapidly commute the purpose of Internal and Emergency in-patient wards.

Unfortunately, the crisis is not over and we are now facing the urgent need for a gradual 'return strategy' that has to include the maintenance of Covid-dedicated areas in the hospital. Again, we know that bad times could come back again and that the world will not be the same anymore. The overwhelming certainty about the great value of this experience will continue to encourage us.
Acknowledgements The medical and nurse staff for their day-by-day, huge, and passionate work and Daniela Santi for the contribution in the editing of this letter.

Author contributions CC, ES and AMB takes responsibility for all aspects of the reliability and freedom from bias of the data presented and their discussed interpretation.

Funding None.

\section{Compliance with ethical standards}

Conflict of interest The authors declare that they have no conflict of interest none.

Statement of human and animal rights statement All procedures followed were in accordance with the ethical standards of the responsible committee on human experimentation (institutional and national) and with the Helsinki Declaration.

Informed consent All study participants provided informed consent prior to their participation.

\section{References}

1. Onder G, Rezza G, Brusaferro S (2020) Case-fatality rate and characteristics of patients dying in relation to COVID-19 in Italy. JAMA. https://doi.org/10.1001/jama.2020.4683. (Published online March 23, 2020)

2. Margutti E, Greco I, Brambilla AM, Maraffi T, Cosentini R (2017) Non-invasive ventilation in acute respiratory failure: the key "W" questions. Intern Emerg Med 12:1307-1311. https://doi. org/10.1007/s11739-017-1721-x

3. Siegel JD, Rhinehart E, Jackson M, Chiarello L, and the Healthcare Infection Control Practices Advisory Committee (2007) Guideline for isolation precautions: preventing transmission of infectious agents in healthcare settings. https://www.cdc.gov/infec tioncontrol/guidelines/isolation/index.html. July 2019

Publisher's Note Springer Nature remains neutral with regard to jurisdictional claims in published maps and institutional affiliations. 